\title{
Impacto econômico das ações judiciais para o fornecimento de medicamentos no Estado de São Paulo
}

\author{
Economic impact of lawsuits for drug supply in the State of São Paulo
}

Impacto económico de las demandas por suministro de drogas en el Estado de São Paulo

\author{
Adriane Lopes Medeiros Simone ${ }^{1}$ \\ Daniela Oliveira de Melo²
}

\section{Resumo}

Objetivos: compreender a participação das despesas com medicamentos judicializados nas despesas totais liquidadas com medicamentos (DTLM) no Estado de São Paulo (SP). Métodos: realizou-se análise exploratória de dados do Sistema de Informações Gerenciais da Execução Orçamentária (Sigeo) e do Sistema de Informação de Orçamento Público em Saúde (Siops), de 2010 a 2018. Resultados: no período estudado, as DTLM em SP superaram 11 bilhões de reais, apresentando tendência decrescente em valores globais e na participação percentual em relação à despesa total com saúde (de 11,32\% em 2010 para 8,95\% em 2018). De 2016 a 2018, 17\% das DTLM foram destinadas às ações judiciais (R\$ $679.935 .967,31$ ), sendo a tendência crescente. Discussão: a representatividade dos medicamentos judicializados na DTLM alerta para questões de custo, mas também para a segurança do paciente e sustentabilidade das políticas públicas de saúde, pois o rol também contempla medicamentos novos, para os quais a superioridade em efetividade e segurança ainda não foram comprovadas comparando-se às alternativas disponíveis, e/ou com uso offlabel. Conclusões: as demandas judiciais por medicamentos não têm levado ao aumento da destinação de recursos para sua aquisição, mas à concorrência por recursos originalmente destinados à política de assistência farmacêutica, com redução da participação percentual dos medicamentos das listas oficiais do Sistema Único de Saúde (SUS) a cada ano.

Palavras-chave: Custos de medicamentos. Custos e análise de custo. Judicialização da saúde. Assistência farmacêutica.

\footnotetext{
Abstract

Objective: to understand the participation of expenses with judicialized medicines in total expenditure on drugs (DTLM) in São Paulo State (SP). Methods: it was conducted an exploratory analysis of data from Sistema de Informações Gerenciais da Execução Orçamentária (Sigeo) and Sistema de Informação de Orçamento Público em Saúde (Siops) from 2010 to 2018. Results: in this period, DTLM in SP exceeded 11 billion of reais, showing a downward trend in global values and percentage share in relation to total health expenditure

${ }^{1}$ Doutora em Saúde Pública; farmacêutica da Coordenadoria de Assistência Farmacêutica; professora contratada III do Departamento de Política, Gestão e Saúde, da Faculdade de Saúde Pública da Universidade de São Paulo, São Paulo, SP, Brasil. E-mail: almedeiros@usp.br

2 Doutora, docente adjunta do Instituto de Ciências Ambientais, Químicas e Farmacêuticas e coordenadora do Núcleo de Avaliação de Tecnologias em Saúde (NATS) da Universidade Federal de São Paulo, Campus Diadema, São Paulo, SP, Brasil. E-mail: melo.daniela@unifesp.br
} 
(from $11.32 \%$ in 2010 to $8.95 \%$ in 2018). From 2016 to $2018,17 \%$ of DTLM were destined to lawsuits ( $\mathrm{R} \$ 679,935,967.31)$, with a growing trend. Discussion: the representativeness of judicialized drugs in DTLM warns of cost issues, but also for patient safety and sustainability of public health policies, because this list also includes new drugs, which have not been proved superior in effectiveness and safety yet, comparing to available alternatives, and/or off label use. Conclusions: the legal demands for medicines have not led to an increase in the allocation of funds for their purchase, but to competition for resources originally destinated for the pharmaceutical services policy, with a reduction in the percentage participation of medicines from SUS official lists each year.

Keywords: Drug costs. Costs and cost analysis. Health judicialization. Pharmaceutical services.

\section{Resumen}

Objetivos: comprender la participación de los gastos con drogas judicializadas en los gastos totales con drogas (DTLM) en el Estado de São Paulo (SP). Métodos: se realizó un análisis exploratorio de datos del Sistema de Informações Gerenciais da Execução Orçamentária (Sigeo) y el Sistema de Informação de Orçamento Público em Saúde (Siops) de 2010 a 2018. Resultados: en este período, DTLM en SP superó los 11 mil millones de reales, mostrando una tendencia a la baja en los valores globales y la participación porcentual en relación con el gasto total en salud (del 11.32\% en 2010 al 8.95\% en 2018). De 2016 a 2018, el 17\% de DTLM se destinó a estas demandas ( $R \$ 679,935,967.31)$, con una tendencia creciente. Discusión: la representatividad de los medicamentos judicializados en DTLM advierte sobre los costos, pero también sobre la seguridad del paciente y la sostenibilidad de las políticas de salud pública, porque esta lista también incluye nuevos medicamentos, que aún no han demostrado ser superiores en comparación de efectividad y seguridade a alternativas disponibles, y/o uso off-label. Conclusiones: los pleitos de medicamentos no han llevado a un aumento en la asignación de fondos para su compra, sino a la competencia por los recursos originalmente destinados a la política de asistencia farmacéutica, con una reducción en el porcentaje de participación de medicamentos de las listas oficiales del SUS cada año.

Palabras-Ilave: Costos de los medicamentos. Costos y análisis de costo. Judicialización de la salud. Servicios farmacéuticos.

\section{Introdução}

Com a população brasileira estimada em 210 milhões de habitantes (1), o Sistema Único de Saúde (SUS) é reconhecido pela Organização Mundial de Saúde (OMS) como um dos maiores sistemas públicos de saúde do mundo (2). Pautado nos princípios de universalidade, equidade e integralidade, o SUS é essencial para a garantia do direito constitucional à saúde $(3,4)$, e contribuiu significativamente para a melhora na condição de saúde da população desde sua implementação, apesar das dimensões continentais do nosso país e da persistência de desigualdades sociais, econômicas e regionais (5).

A Constituição Federal de 1988 determina que a União, os Estados, Distrito Federal 
e os Municípios são corresponsáveis pelas despesas com saúde, aplicadas as regras de financiamento mínimo da receita de impostos para Estados e Municípios (3). De acordo com o Sistema de Informação de Orçamento Público em Saúde (Siops), entre 2010 e 2018, a participação da receita própria aplicada em saúde no Estado de São Paulo foi, em média, $12,82 \%$ (6), valor um pouco acima do estabelecido pela legislação (7). Durante esse período, foram investidos 949,5 bilhões de reais em saúde, considerando-se tanto os recursos próprios deste Estado quanto os valores transferidos pelo Ministério da Saúde, mediante definição constitucional (6).

Por figurar um componente essencial do sistema de saúde em todos os níveis de complexidade, a política de assistência farmacêutica é integrante da política de saúde (4). Assim, no processo de gestão do SUS, a estruturação da assistência farmacêutica, a promoção do acesso e do uso racional de medicamentos correspondem a responsabilidades solidárias dos entes federativos (8).

O acesso integral à assistência farmacêutica compreende a dispensação de medicamentos e produtos de interesse para a saúde, em conformidade com as diretrizes terapêuticas e com as relações de medicamentos instituídas pelos gestores federal, estadual e municipal, bem como a oferta de procedimentos terapêuticos, em regime domiciliar, ambulatorial e hospitalar, constantes de tabelas elaboradas pelo gestor federal do SUS (9).

Sabe-se que a propositura de ações judiciais relacionadas ao direito à saúde, contra os poderes públicos, tornou-se um dos maiores desafios para gestores de saúde na atualidade, inclusive no campo da assistência farmacêutica (10-12). Nos últimos anos, houve um aumento quase exponencial do número de ações judiciais e dos valores empregados no atendimento a essas demandas, o que tem sido discutido como o fenômeno da judicialização, atingindo simultaneamente a União, os Estados, o Distrito Federal (DF) e os Municípios (10,12-15). Entre os anos de 2008 e 2015, apenas os gastos da União com a oferta de medicamentos devido a ações judiciais passaram de 70 milhões a 1 bilhão de reais (16). No Estado de São Paulo, a maioria das ações judiciais contra a Secretaria de Estado da Saúde de São Paulo (SES/SP) reivindica o acesso a medicamentos (12) e, em 2018, o valor liquidado para aquisição de medicamentos para o atendimento dessas ações superou 355 milhões de reais, valor que ainda pode estar subestimado (17).

Os estudos sobre a judicialização em saúde têm analisado tanto as características 
das demandas quanto dos medicamentos judicializados. Também tem analisado a evolução do volume de gastos associados às demandas judiciais como forma de discutir seu impacto no planejamento e na execução das políticas públicas na área da saúde e/ou impacto nos princípios de universalidade e equidade do SUS $(10-14,18,19)$. Diante do cenário já bastante conhecido e discutido, ainda é importante elucidar o impacto das aquisições de medicamentos de ações judiciais na despesa com saúde; se esse gasto público é sustentável; e seu potencial de interferir no acesso a medicamentos essenciais pela população (20).

\section{Metodologia}

Com o objetivo de compreender a participação das despesas com medicamentos em ações judiciais no total de despesas com medicamentos no Estado de São Paulo, este estudo de caso apresenta a análise exploratória de dados secundários do Sistema de Informações Gerenciais da Execução Orçamentária (Sigeo), disponibilizados pela Secretaria de Estado da Fazenda, em atendimento à Lei de Acesso à Informação (18).

O Sigeo é um sistema de informação que possibilita a transparência dos gastos públicos, por meio da elaboração de relatórios de informações da execução orçamentária e financeira dos órgãos e entidades da administração direta e indireta do Estado, a partir de 2010, com detalhamento das despesas empenhadas, liquidadas e pagas - do ano e de restos a pagar, incluídas as intra-orçamentárias. Os dados são extraídos do Sistema Integrado de Administração Financeira para Estados e Munícipios (Siafem/SP) e atualizados diariamente (17).

Os relatórios do Sigeo foram extraídos em 24 de julho de 2019, utilizando os seguintes filtros: i) Exercício: 2010 a 2018; ii) Fase: liquidado, conforme previsto no art. 63 da Lei no 4.320/1964; iii) Programa do Plano Plurianual: 0930 - atendimento integral e descentralizado no SUS/SP; iv) Ação do plano plurianual: em 2010 e 2011, 48500000 - atendimento ambulatorial e hospitalar em unidade estadual; e a partir de 2012, 61170000 - assistência farmacêutica especializada.

Nesses relatórios, foram identificadas as despesas liquidadas com a aquisição de medicamentos, a partir dos subelementos de despesa 33903030-medicamentos e insumos farmacêuticos e 33903035 - medicamentos fornecidos por decisão judicial (17), como 
desdobramento do elemento de despesa 30 - material de consumo, conforme detalhamento do Manual Técnico do Orçamento 2018, $7^{\mathfrak{a}}$ versão (21). A segregação do subelemento de despesa 33903035 - medicamentos fornecidos por decisão judicial se deu somente a partir de 2016 (17).

De forma complementar, foi extraída a série histórica do indicador participação percentual da despesa com medicamentos na despesa total com saúde pelo Siops, baseada nas informações transmitidas pela SES/SP até julho/2019, empregando os filtros: i) Estados e DF: série história de indicadores; ii) Linha: UF; iii) Coluna: Ano; iv) Conteúdo: 2.3 \%D.Medicamt/DT; v) Períodos disponíveis: 2010 a 2018; e vi) UF: São Paulo (6).

A transmissão de dados por meio do Siops é de natureza declaratória e obrigatória para União, Estados, Distrito Federal e Municípios, conforme Lei Complementar nº141/2012, entretanto, admite-se que os entes federativos corrijam os dados relativos a um exercício financeiro, se necessário. Estas características relativas à natureza e operação do SIOPS têm duas implicações: os valores apresentados podem estar subestimados, ainda que em pequenas proporções, e as comparações de valores resultantes de buscas em diferentes momentos são limitadas $(22,23)$.

\section{Resultados e discussão}

Como pode ser observado no gráfico 1, no período analisado, de 2010 a 2018, as despesas totais liquidadas com medicamentos somaram um valor superior a 11 bilhões de reais $(R \$ 11.873 .426 .052,00)$. Em geral, houve pequenas variações anuais: - $3 \%, 2011 / 2010$, 2012/2011, 2014/2013; -1\%, 2013/2012, 2015/2014; -0,2\%, 2016/2015; e 0,5\%, 2018/2017, com exceção de 2017, quando a despesa total liquidada foi $13 \%$ superior à 2016 , apesar de se manter inferior ao valor de 2010. Ainda, a participação percentual da despesa com medicamentos na despesa total com saúde no Estado de São Paulo apresentou tendência decrescente na maior parte do tempo e passou de 11,32\%, em 2010, para 8,95\%, em 2018. Portanto, no período analisado, as despesas totais liquidadas com medicamentos apresentaram tendência decrescente tanto em valores, quanto em participação percentual em relação à despesa total com saúde, com sinais de recuperação nos últimos três anos para o montante total, mas não para a participação percentual. Isso demonstra que, apesar de se manter superior à participação percentual dos demais Estados brasileiros (6), o valor 
empenhado para aquisição de medicamentos tem sofrido a influência da competição do montante com outras tecnologias ou serviços de saúde.

Gráfico 1. Evolução da despesa com medicamentos no Estado de São Paulo e participação percentual dos medicamentos fornecidos para ações judiciais, de 2010 a 2018

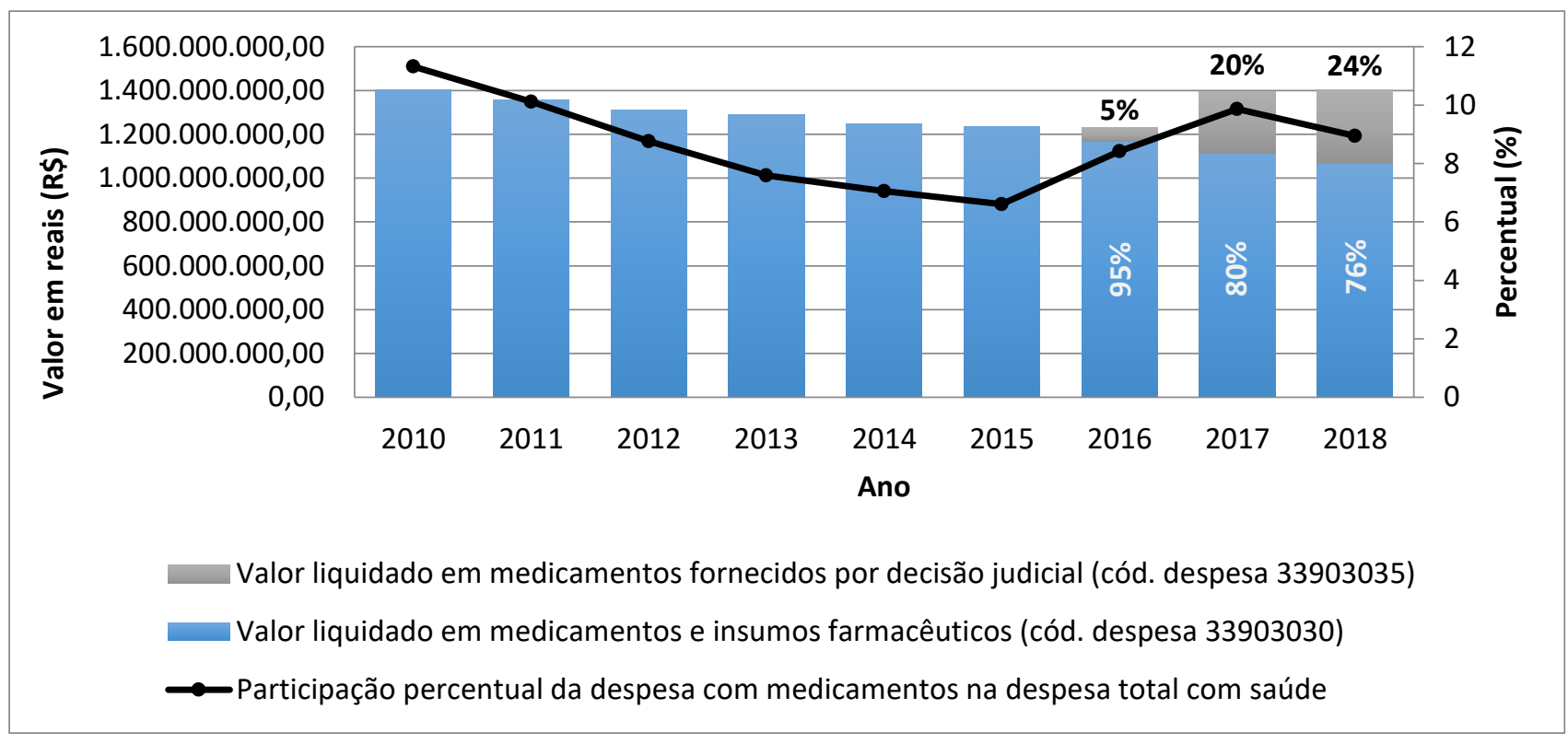

Fonte: (6), (17)

O percentual da despesa liquidada com medicamentos dos Programas Oficiais de Assistência Farmacêutica - aqueles para os quais há programação de fornecimento pelo Estado de São Paulo - e para o atendimento das ações judiciais, ano a ano, também foi representado, considerando-se a disponibilidade da informação. Verificou-se que, das despesas totais liquidadas com medicamentos no valor de $\mathrm{R} \$ 4.032 .328 .810,94$ no período de 2016 a 2018, 17\% foram destinadas aos medicamentos adquiridos em decorrência de decisão judicial ( $R \$ 679.935 .967,31)$, sendo a tendência crescente. Cabe ressaltar que a mudança recente no formato de execução orçamentária em âmbito estadual, com a utilização do subelemento de despesa 33903035 - medicamentos fornecidos por decisão judicial a partir de 2016 -, pode ter levado a valores subestimados referentes à aquisição de medicamentos por decisão judicial.

É importante destacar que, nas demandas por medicamentos, estão incluídas tecnologias com a devida comprovação de eficácia e segurança, registradas junto à Agência 
Nacional de Vigilância Sanitária (Anvisa), incorporados ou não no SUS, mas também medicamentos novos, com ou sem registro sanitário no país. São medicamentos recentemente introduzidos na prática clínica e para os quais ainda não foi devidamente comprovada a superioridade em efetividade e/ou a segurança, comparando-se com as alternativas terapêuticas disponíveis e/ou que estão sendo usadas fora das indicações para as quais foram registradas, chamado de uso off-label $(10,11,13,19,24)$. Portanto, não é apenas uma questão de custo, mas também de segurança do paciente e de sustentabilidade das políticas públicas de saúde.

Em um estudo semelhante realizado no Estado de Alagoas, foi avaliada a participação dos medicamentos nas despesas totais em saúde e a aquisição de medicamentos judicializados frente ao total gasto com medicamentos entre 2010 e 2017 (15). Enquanto o atendimento às demandas judiciais resultou em participação percentual nas despesas totais liquidadas com medicamentos de 10,3\%, em 2017, em Alagoas (15); no Estado de São Paulo chegou a $20 \%$, em 2017, e $24 \%$, em 2018, o que sugere maior impacto da judicialização para obtenção de medicamentos no Estado de São Paulo.

\section{Conclusão}

Considerando não ter havido incremento nas despesas totais liquidadas com medicamentos no Estado de São Paulo, é possível concluir que as demandas judiciais por medicamentos não têm levado ao aumento da destinação de recursos para sua aquisição o que permitiria o preenchimento de lacunas da Política de Assistência Farmacêutica evidenciadas pela judicialização, mas à concorrência por recursos originalmente destinados aos programas de assistência farmacêutica, com redução da participação percentual dos medicamentos das listas oficiais do SUS a cada ano.

\section{Referências}

1. Instituto Brasileiro de Geografia e Estatística. Projeção da população do Brasil e das Unidades da Federação. Disponível em: https://www.ibge.gov.br/apps/populacao/projecao/ [Acesso 29 jul. 2019]. 
2. Organização Panamericana de Saúde. Relatório 30 anos de SUS. Que SUS para 2030? Versão preliminar [Internet]. Brasília - DF; 2018. Disponível em: https://apsredes.org/wpcontent/uploads/2018/10/Serie-30-anos-001-SINTESE.pdf [Acesso 29 jul. 2019].

3. Brasil. Constituição (1988). Constituição da República Federativa do Brasil. Brasília - DF: Senado; 1988.

4. Brasil. Lei n 8.080 , de 19 de setembro de 1990. Dispõe sobre as condições para a promoção, proteção e recuperação da saúde, a organização e o funcionamento dos serviços correspondentes e dá outras providências. Diário Oficial da União, Brasília, DF; 20 set 1990.

5. Castro MC, Massuda A, Almeida G, Menezes Filho NA, Andrade MV, Noronha KVMS, et al. Brazil's unified health system: the first 30 years and prospects for the future. Lancet [Internet]. 2019 Jul;394(10195):345-56.

6. Brasil. Sistema de Informação de Orçamento Público em Saúde [Internet]. Brasília - DF: Departamento de Informática do SUS; 2017. Disponível em: http://siops.datasus.gov.br/ [Acesso 28 jul. 2019].

7. Brasil. Emenda Constitucional $n^{\circ} 29$, de 13 de setembro de 2000. Altera os arts. 34, 35, 156, 160, 167 e 198 da Constituição Federal e acrescenta artigo ao Ato das Disposições Constitucionais Transitórias, para assegurar os recursos mínimos para o financiamento das ações e serviços públicos de saúde. Diário Oficial da União. 14 set. 2000.

8. Brasil. Resolução $n^{\circ} 4$, de 19 de julho de 2012. Dispõe sobre a pactuação tripartite acerca das responsabilidades sanitárias no âmbito do Sistema Único de Saúde (SUS), para fins da transição entre o Pacto pela Saúde e a sistemática do Contrato Organizativo de Ação Pública de Saúde (COAP). Diário Oficial da União, Brasília, DF; 2012.

9. Brasil. Lei no 12.401, de 28 de abril de 2011. Altera a Lei no 8.080, de 19 de setembro de 1990, para dispor sobre a assistência terapêutica e a incorporação de tecnologia em saúde no âmbito do Sistema Único de Saúde - SUS. Diário Oficial da União, Brasília, DF, 2011.

10. Pepe VLE, Figueiredo TA, Simas L, Osorio-de-Castro CGS, Ventura M. A judicialização da saúde e os novos desafios da gestão da assistência farmacêutica. Cien Saude Colet. 2010 Aug;15(5):2405-14.

11. Biehl J, Socal MP, Amon JJ. The Judicialization of Health and the Quest for State Accountability: Evidence from 1,262 Lawsuits for Access to Medicines in Southern Brazil. Health Hum Rights. 2016 Jun;18(1):209-20.

12. Chieffi AL, Barradas RDCB, Golbaum M. Legal access to medications: a threat to Brazil's public health system? BMC Health Serv Res. 2017;17(1):499. 
13. Machado MAA, Acurcio FA, Brandão CMR, Faleiros DR, Guerra AA, Cherchiglia ML, et al. Judicialization of access to medicines in Minas Gerais state, Southeastern Brazil. Rev Saude Publica. 2011 Jun;45(3):590-8.

14. Stamford A, Cavalcanti M. Legal decisions on access to medicines in Pernambuco, Northeastern Brazil. Rev Saude Publica. 2012 Oct;46(5):791-9.

15. Silva RL, Santos JJ, Santa Rita LP, Pinto IMBS. Judicialização e Políticas Públicas: o impacto do fornecimento de medicamentos por determinação judicial no orçamento da Política de Saúde do Estado de Alagoas (2010-2017). Rev Adm Roraima - RARR. 2018 Dec 28;8(2):326.

16. Brasil. Tribunal de Contas da União (TCU). Decisão 1787/2017. [Internet]. 2017 [Acesso 2 agosto 2019]. Disponível em: https://portal.tcu.gov.br/imprensa/noticias/\%0Aaumentam-os-gastos-publicos-comjudicializacao-da-saude.htm

17. Sistema de Informações Gerenciais da Execução Orçamentária. São Paulo (Brasil): Secretaria da Fazenda. 2019. Disponível em: https://www.fazenda.sp.gov.br/SigeoLei131/Paginas/FlexConsDespesa.aspx [Acesso 28 julho 2019].

18. Pandolfo M, Delduque MC, Amaral RG. Legal and sanitary aspects conditioning access to medicines in Brazilian courts. Rev Salud Publica (Bogota). 14(2):340-9.

19. Biehl J, Amon JJ, Socal MP, Petryna A. Between the court and the clinic: lawsuits for medicines and the right to health in Brazil. Health Hum Rights. 2012 Jun 15;14(1):E36-52.

20. Medeiros AL. Assistência farmacêutica no Sistema Único de Saúde. Responsabilidade compartilhada por União, Estado e Municípios. 2018. 260 p. Tese (Doutorado em Ciências) - Faculdade de Saúde Pública, Universidade de São Paulo, São Paulo, 2018.

21. Brasil. Ministério do Planejamento, Desenvolvimento e Gestão. Secretaria de Orçamento Federal. Manual Técnico de Orçamento - MTO. Edição 2018. Brasília; 2017. Disponível em:

https://www1.siop.planejamento.gov.br/mto/lib/exe/fetch.php/mto2018:mto_atual.pdf [Acesso 28 julho 2019].

22. Vieira FS, Zucchi P. Financiamento da assistência farmacêutica no sistema único de saúde. Saúde e Soc. 2013 Mar;22(1):73-84. Disponível em: http://www.scielo.br/scielo.php?script=sci_arttext\&pid=S0104$12902013000100008 \&$ Ing=pt\&tIng=pt [Acesso 28 julho 2019].

23. Brasil. Lei complementar $n^{\circ} 141$, de 13 de janeiro de 2012. Regulamenta o $\S 30$ do art. 198 da Constituição Federal para dispor sobre os valores mínimos a serem aplicados anualmente pela União, Estados, Distrito Federal e Municípios em ações e serviços 
públicos de saúde; estabelece os critérios de rateio dos recursos de transferências para a saúde e as normas de fiscalização, avaliação e controle das despesas com saúde nas 3 (três) esferas de governo; revoga dispositivos das Leis nos 8.080, de 19 de setembro de 1990, e 8.689, de 27 de julho de 1993; e dá outras providências. Diário Oficial da União, Brasília, DF; 16 jan 2012.

24. Ferraz OLM. The right to health in the courts of Brazil: worsening health inequities? Health Hum Rights. 2009;11:33-45.

Como citar esse artigo:

Simone ALM, Melo DO. Impacto econômico das ações judiciais para o fornecimento de medicamentos no Estado de São Paulo. Cadernos Ibero-Americanos de Direito Sanitário. 2019 jul./set.; 8(3): 60-69.

http://dx.doi.org/10.17566/ciads.v8i3.565 